\title{
MiR-182-5p Knockdown Targeting PTEN Inhibits Cell Proliferation and Invasion of Breast Cancer Cells
}

\author{
Yue-Sheng Zhao' ${ }^{1}$, Wei-Chao Yang ${ }^{2}$, Hong-Wei Xin ${ }^{3}$, Ji-Xia Han ${ }^{3}$, and Su-Gang Ma \\ ${ }^{1}$ Department of Breast Surgery, The Third Hospital Affiliated to Qiqihar Medical College, Qiqihar; \\ ${ }^{2}$ Department of Breast Surgery, Jinan Zhangqiu District Hospital of Traditional Chinese Medicine, Zhangqi District, Jinan; \\ ${ }^{3}$ Department of General Surgery, Sixth People's Hospital of Ji'nan City, Jinan; \\ ${ }^{4}$ Department of Breast Surgery, Sixth People's Hospital of Ji'nan City, Jinan, China
}

\begin{abstract}
Purpose: Breast cancer (BC) is one of the most common malignant tumors, affecting a significant number of women worldwide. MicroRNAs (miRNAs) have been reported to play important roles in tumorigenesis. The aim of this study was to determine the roles of miR-182-5p in BC progression.

Materials and Methods: The expressions of miR-182-5p and phosphatase and tensin homolog deleted on chromosome 10 (PTEN) were measured in BC tissues and cells by quantitative real-time polymerase chain reaction or Western blot. Cell proliferation and invasion were detected by cell counting kit-8 assay and trans-well assay, respectively. The interaction between miR-182$5 \mathrm{p}$ and PTEN was probed by bioinformatics analysis, luciferase activity, and RNA immunoprecipitation. A murine xenograft model was established to investigate the role of miR-182-5p in BC progression in vivo.

Results: An abundance of miR-182-5p was noted in BC tissues and cells. High expression of miR-182-5p was associated with poor survival. Abrogation of miR-182-5p inhibited cell proliferation and invasion in BC cells. Interestingly, PTEN was indicated as a target of miR-182-5p, and its restoration reversed miR-182-5p-mediated promotion of proliferation and invasion of BC cells. Moreover, depletion of miR-182-5p suppressed tumor growth via up-regulating PTEN expression in the murine xenograft model. Conclusion: MiR-182-5p exhaustion blocked cell proliferation and invasion by regulating PTEN expression, providing a novel therapeutic avenue for treatment of BC.
\end{abstract}

Key Words: Breast cancer, miR-182-5p, PTEN, proliferation, invasion

\section{INTRODUCTION}

Breast cancer (BC) is a common malignant tumor and threatens a significant number of women worldwide. ${ }^{1}$ Invasion contributes to the tumor's malignancy and the high incidence of BC in older adult women. ${ }^{2}$ Although technology for diagnosis and therapy is advancing, no approved treatment has proven completely effective. ${ }^{3}$ Therefore, a novel driver for di-

Received: August 14, 2018 Revised: October 31, 2018

Accepted: November 4, 2018

Corresponding author: Su-Gang Ma, MB, Department of Breast Surgery, Sixth People's Hospital of Ji'nan City, No. 1920, Huiquan road, Zhangqiu District, Ji'nan City, Shandong Province 250200, China

Tel: 86-452-269-7522, E-mail: shdlkajhdk750@126.com

-The authors have no potential conflicts of interest to disclose.

(C) Copyright: Yonsei University College of Medicine 2019

This is an Open Access article distributed under the terms of the Creative Commons Attribution Non-Commercial License (https://creativecommons.org/licenses/ by-nc/4.0) which permits unrestricted non-commercial use, distribution, and reproduction in any medium, provided the original work is properly cited. agnosis and therapeutics of $\mathrm{BC}$ is required.

MicroRNAs (miRNAs) are a class of small noncoding RNAs and participate in tumorigenic processes in various cancers, including BC. ${ }^{4}$ Dysregulation of miRNAs has been suggested to be associated with cell apoptosis, proliferation, invasion, and metastasis of cancers in $\mathrm{BC}{ }^{5}$ For instance, overexpression of miR-18a-5p was shown to inhibit cell migration, invasion, and epithelial-mesenchymal transition by targeting sterol regulatory element binding transcription protein 1 (SREBP1) in BC. ${ }^{6}$ MiR-185-5p inhibited invasion, migration, and epithelialmesenchymal transition by regulating receptors for advanced glycosylation end-product specific receptor (RAGE) in BC. ${ }^{7}$ In addition, miR-19b was found to contribute to cell proliferation, migration, and invasion by mediating phosphatidylinositide 3-kinase (PI3K) and serine-threonine kinase (Akt) pathways, serving as a prognostic biomarker of BC. ${ }^{8}$ Moreover, miR-30 family members have been reported to play a pivotal role in cell invasion in BC. ${ }^{9}$ Apart from these miRNAs, serum miR- 
1915-3p and miR-455-3p have also been implicated in BC progression by regulating cell proliferation and migration ability. ${ }^{10}$

Research has demonstrated that miR-182-5p protects cell viability, migration, and invasion by regulating rat sarcoma (RAS)-associated binding 27a (RAB27A) in gastric cancer cells. ${ }^{11}$ In addition, miR-182-5p has been shown to promote cell invasion and proliferation in prostate cancer through mediating forkhead box F2 (FOXF2), reversion-inducing-cysteine-rich protein with Kazal motifs (RECK) and metastasis suppressor 1 (MTSS1). ${ }^{12}$ Notably, miR-182-5p has been suggested to be ectopic in patients with $\mathrm{BC} .{ }^{13}$ Also, inhibition of miR-182-5p has been found to elicit pro-apoptosis and antiproliferation effects via regulating Caspase 9 expression in BC. ${ }^{14}$ However, the role of miR-182-5p in BC and its mechanism are not fully understood.

Phosphatase and tensin homolog deleted on chromosome 10 (PTEN) has been suggested as a tumor suppressor, leading to diverse biological outcomes by regulating metabolism and growth in varying cancers. ${ }^{15}$ PTEN has been reported to be impaired in multiple cancers and to play an important role in cell proliferation and migration in BC. ${ }^{16}$ Likewise, miR-142-5p also suppresses PTEN expression to further modulate cell proliferation and apoptosis in BC cells. ${ }^{17}$ These results suggest that PTEN opens up the possibility of treatment of BC. However, there is no direct evidence in support of the relationship between miR-182-5p and PTEN. In this study, we investigated the effect of miR-182-5p on cell proliferation, invasion, and tumor growth in vivo and examined the interaction between miR-182-5p and PTEN in BC cells.

\section{MATERIALS AND METHODS}

\section{Specimens}

This research was approved by the Institutional Research Ethics Committee of the Third Hospital Affiliated to Qiqihar Medical College, and written informed consent was obtained from all patients. In this study, a total of $37 \mathrm{BC}$ tissues and paired adjacent non-cancer tissues (NC) were collected from patients without the history of radiotherapy or chemotherapy before operation and stored in liquid nitrogen until ready for use. Patients were classified into high $(n=22)$ and low miR182-5p expression groups $(n=15)$ according to the mean value of miR-182-5p abundance. The 5-year survival rate assay was conducted in all participants. The clinicopathologic characteristics of BC patients are displayed in Table 1.

\section{Cell culture and transfection}

The normal breast epithelial cell line MCF-10A and BC cell lines MCF-7, MDA-MB-231, BT20, T47D, and SKBR3 were obtained from American Tissue Culture Collection (ATCC, Manassas, VA, USA). All cells were cultured in Dulbecco's Modified Eagle Medium (Gibco, Carlsbad, CA, USA) contain-
Table 1. Relationship between miR-182-5p Expression and Clinicopathologic Characteristics of Breast Cancer Patients

\begin{tabular}{|c|c|c|c|c|}
\hline \multirow{2}{*}{ Characteristics } & \multirow{2}{*}{ Cases } & \multicolumn{2}{|c|}{ miR-182-5p expression } & \multirow{2}{*}{$p$ value } \\
\hline & & Low, n (\%) & High, n (\%) & \\
\hline Age (yr) & & & & 0.7312 \\
\hline$\leq 40$ & 9 & $4(44.44)$ & $5(55.56)$ & \\
\hline$>40$ & 28 & 11 (39.29) & $17(60.71)$ & \\
\hline Tumor size (cm) & & & & 0.7028 \\
\hline$<2$ & 8 & $3(37.50)$ & $5(62.50)$ & \\
\hline$\geq 2$ & 29 & $12(41.38)$ & $17(58.62)$ & \\
\hline Differentiation & & & & 0.3251 \\
\hline Well & 3 & $1(33.33)$ & $2(66.67)$ & \\
\hline Moderate & 6 & $3(50.00)$ & $3(50.00)$ & \\
\hline Poor & 28 & $11(39.29)$ & $17(60.71)$ & \\
\hline Lymph node metastasis & & & & 0.0396 \\
\hline 0 & 3 & 1 (33.33) & 2 (66.67) & \\
\hline $1-3$ & 8 & $4(50.00)$ & $4(50.00)$ & \\
\hline$>3$ & 26 & $10(38.46)$ & 16 (61.54) & \\
\hline Tumor stage & & & & 0.0237 \\
\hline$|+| \mid$ & 13 & $6(46.15)$ & $7(53.85)$ & \\
\hline$\|+1 \mathrm{IV}$ & 24 & $9(37.50)$ & $15(62.50)$ & \\
\hline
\end{tabular}

ing $10 \%$ fetal bovine serum (Gibco), $1 \%$ penicillin, and streptomycin (Invitrogen, Carlsbad, CA, USA) at $37^{\circ} \mathrm{C}$ in $5 \% \mathrm{CO}_{2}$ during the study.

PTEN overexpression vectors (PTEN) were cloned into pcDNA by GenePharma (Shanghai, China). MiR-182-5p mimics (miR182-5p), miR-182-5p inhibitor (anti-miR-182-5p), and negative control (miR-NC or anti-NC) were obtained from GenePharma. To investigate the effect of miR-182-5p and PTEN on cell proliferation and invasion, transfection was performed in MCF-7 and MDA-MB-231 cells using Lipofectamine 2000 (Invitrogen) according to the manufacturer's protocol.

\section{Quantitative real-time polymerase chain reaction}

TRIzol reagent (Invitrogen) was used to isolate total RNA from tissues or cells following the manufacturer's instructions. The quality of RNA from every group was evaluated with a NanoDrop Spectrophotometer (NanoDrop, Wilmington, DE, USA), followed by synthesis of complementary DNA (cDNA) using a commercial Reverse Transcription Kit (Applied Biosystems, Foster City, CA, USA). In turn, synthesized cDNA was diluted for quantitative real-time polymerase chain reaction (qRT-PCR) with SYBR Green Real-time PCR Master Mix (Toyobo, Tokyo, Japan) following the amplification instructions. All primers were listed as follows: miR-182-5p (Forward, 5'-TGCGGTTTG GCAATGGTAGAAC-3'; Reverse, 5'-CCA GTGCAGGGTCCGAG GT-3'), U6 (Forward, 5'-CTCGCTTCGGCAGCACA-3'; Reverse, 5'-AACGCTTCACGAATTTGCGT-3'), PTEN (Forward, 5'-CCCT TTG AAGACCATAACCCAC-3'; Reverse, 5'-TTACACCAGTTC GTCCCT-3'), $\beta$-actin (Forward, 5'-CAGCCTTCCTTCTTGGG TAT-3'; Reverse, 5'-TGGCATAGAGGTC TTTACGG-3'). The ex- 
pression of miR-182-5p or PTEN was analyzed by the $2^{-\Delta \Delta C t}$ method using U6 or $\beta$-actin as housekeeping gene, respectively.

\section{Cell proliferation assay}

Cell counting kit-8 (CCK-8) assay was performed to assess cell proliferation in MCF-7 and MDA-MB-231 cells. Cells were seeded into 96-well plates at a density of $1 \times 10^{4}$ cells per well overnight. Then transfection was conducted for $24,48,72$, and $96 \mathrm{~h}$. At the end point, CCK-8 solution (Sigma, St. Louis, MO, USA) was added into each well and incubated for another $2 \mathrm{~h}$ at $37^{\circ} \mathrm{C}$. Optical density was measured at $450 \mathrm{~nm}$ with a microplate reader (Bio-Rad, Hercules, CA, USA).

\section{Invasion assay}

Cell invasion was analyzed by trans-well assay. MCF-7 and MDA-MB-231 cells were cultured in the upper chambers (Costar, Corning, NY, USA) pre-coated with Matrigel (BD Biosciences, San Jose, CA, USA) using serum-free medium at $37^{\circ} \mathrm{C}$ for $24 \mathrm{~h}$ and then carefully removed using a cotton swab. Invasive cells on the basal side of the membrane were fixed with $4 \%$ paraformaldehyde for $10 \mathrm{~min}$, stained with crystal violet (Sigma), and then counted under a microscope (Olympus, Tokyo, Japan). Three visual fields were randomly selected.

\section{Luciferase activity assays}

miRTarBase and miRanda analysis revealed putative binding sites for miR-182-5p and 3' untranslated regions (3'-UTR) sequences of PTEN. The wild-type plasmid (PTEN-WT) or mutant-type plasmid (PTEN-MUT) was generated using pGL3 luciferase reporter vector (Promega, Madison, WI, USA) based on wild or mutant sequences of 3'-UTR of PTEN, respectively. WT or MUT luciferase reporter plasmids, Renilla luciferase plasmid, and miR-182-5p or anti-miR-182-5p were transfected into MCF7 and MDA-MB-231 cells for $48 \mathrm{~h}$ using Lipofectamine 2000 (Invitrogen) according to the manufacturer's protocols. The luciferase activity analysis was evaluated by using Luciferase Assay Kit (Promega) and normalized by Renilla luciferase activity.

\section{RNA immunoprecipitation}

RNA immunoprecipitation (RIP) assay was performed in MCF7 and MDA-MB-231 cells with transfection of miR-182-5p mimics or miR-NC using a Magna RIP RNA-Binding Protein Immunoprecipitation Kit (Millipore, Billerica, MA, USA). Transfected cells were lysed in cell lysis buffer and added to magnetic beads bound with anti-argonaute 1 (Agol) or IgG antibody for $2 \mathrm{~h}$ at $4^{\circ} \mathrm{C}$. The RNA-protein-beads complexes were isolated for qRT-PCR.

\section{Western blots}

Total proteins were isolated from tumor tissues or cells using cell lysis buffer containing $1 \%$ protease inhibitor (Thermo Fisher, Wilmington, DE, USA) and quantified using BCA assay kit (Sigma) according to the instructions. Denatured proteins were separated by SDS-PAGE gel electrophoresis and then transferred to polyvinylidene difluoride membranes (Millipore). The membranes were blocked with blocking reagent (Thermo Fisher) for $1 \mathrm{~h}$ at room temperature and then incubated with primary antibodies against PTEN or $\beta$-actin (Cell Signaling Technology, Danvers, MA, USA) overnight at $4^{\circ} \mathrm{C}$, followed by interacting with horseradish peroxidase conjugated secondary antibodies (Cell Signaling Technology) for 2 $\mathrm{h}$ at room temperature. The protein blots were detected using enhanced chemiluminescence chromogenic substrate (GE Healthcare, Amersham, UK) and investigated by Image Lab software (Bio-Rad).

\section{Animals and MCF-7 xenograft model}

All experiments were approved by the Animal Research committee of the Third Hospital Affiliated to Qiqihar Medical College. SPF BALB/c nude mice (female, 6-week-old) were obtained from Vital River Laboratory Animal Technology (Beijing, China) and maintained in specific pathogen-free microisolator cages with free access to water and food during the experiments.

MCF-7 cells were transfected with lentiviral vectors with miR-182-5p inhibitor (anti-miR-182-5p) or control (anti-NC) constructed by GeneCopoeia (Rockville, MD, USA) according to the manufacturer's instructions. Stably transfected cells were subcutaneously injected into nude mice after acclimatization ( $\mathrm{n}=8$ per group). Tumors were examined every ten days, and tumor volume was calculated with slide calipers as (length $\times$ width $\times$ height) $/ 2$. Mice were sacrificed at 50 days after cell implantation, and tumor specimens were collected for weight or further molecular analyses.

\section{Statistical analysis}

Data are presented as the mean \pm SEM from three independent experiments. Student's $t$ test was used to evaluate differences using SPSS 18.0 software (SPSS, Inc., Chicago, IL, USA). Kaplan-Meier plots were used to analyze survival rates. Multivariate Cox regression analyses were conducted to assess the prognostic value of miR-182-5p and other factors. Statistical significance was considered for $p$ values less than 0.05 .

\section{RESULTS}

\section{MiR-182-5p expression is enhanced in BC tissues and cell lines}

To probe the roles of miR-182-5p in BC progression, its expression was detected in $\mathrm{BC}$ tissues and cell lines. A marked increase in miR-182-5p levels was noted in BC tissues, compared with that in para-tumor samples ( $\mathrm{n}=37$ ) (Fig. 1A). Classifying patients into high miR-182-5p expression $(\mathrm{n}=22)$ and low miR-182-5p expression $(n=15)$ groups according to the mean value thereof, we found that high miR-182-5p expres- 
sion was correlated with poor survival rate $(p<0.01)$ (Fig. 1B). Moreover, high miR-182-5p expression was significantly associated with lymph node metastasis $(p=0.0396)$ and tumor stage ( $p=0.0237$ ). Similarly, an abundance of miR-182-5p was strong higher in MCF-7, MDA-MB-231, BT20, T47D, and SKBR3 cells than in MCF-10A cells, particularly in MCF-7 and MDAMB-231 cells (Fig. 1C). Hence, MCF-7 and MDA-MB-231 cells were used for further experiments.

\section{MiR-182-5p depletion inhibits proliferation and invasion of BC cells}

In the light of dysregulation of miR-182-5p, we further investigated the effect of miR-182 depletion on BC cell proliferation and invasion in MCF-7 and MDA-MB-231 cells with transfection of anti-miR-182-5p or anti-NC. As a result, the expression of miR-182-5p was effectively reduced in MCF-7 and MDAMB-231 cells transfected with anti-miR-182-5p, compared with cells treated with anti-NC (Fig. 2A). Moreover, abrogation of miR-182-5p resulted in a loss of proliferation rate, compared with treatment of anti-NC in MCF-7 and MDA-MB-231
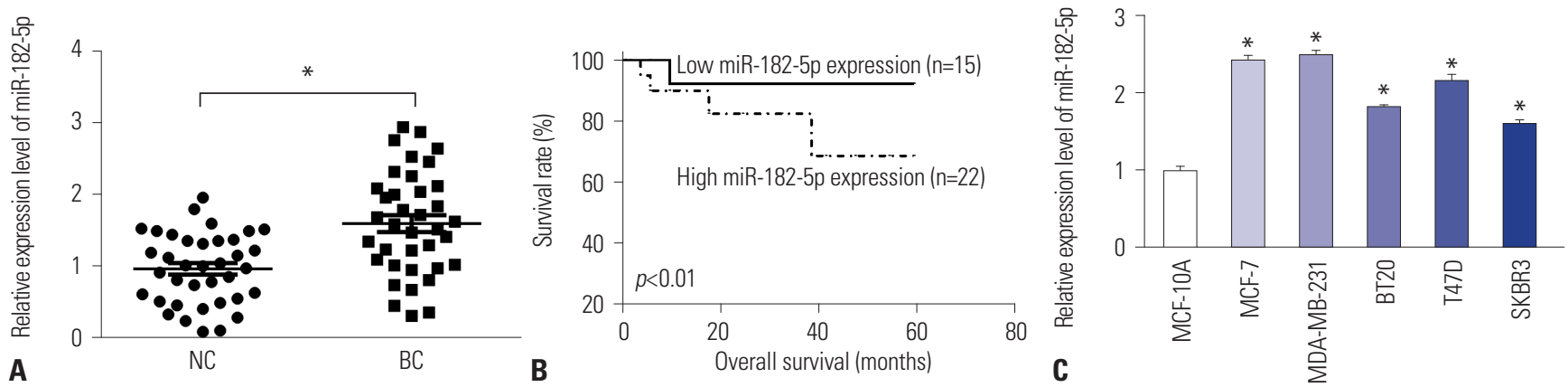

Fig. 1. MiR-182-5p is highly expressed in breast cancer (BC) tissues and cell lines. (A) The abundance of miR-182-5p was examined in BC tissues compared with that in adjacent non-cancer tissues (NC). (B) The survival rate was investigated in BC patients classified according to low miR-182-5p expression and high miR-182-5p expression, $p<0.01$. (C) The expression of miR-182-5p was detected in BC cell lines (MCF-7, MDA-MB-231, BT20, T47D, and SKBR3), compared with that in normal breast epithelial cell line (MCF-10A). * $p<0.05$.

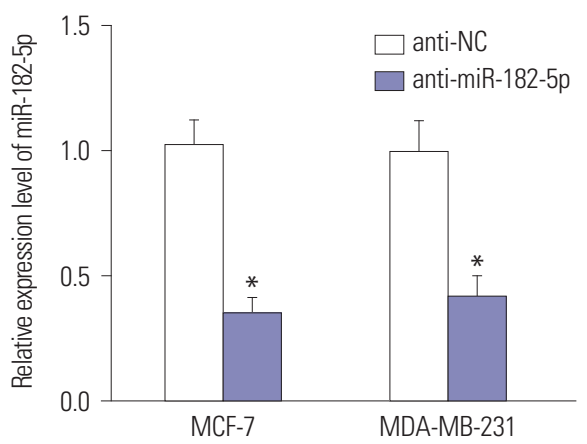

A

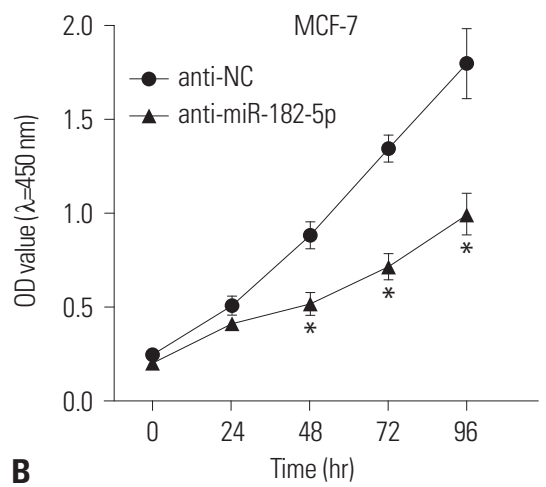

B
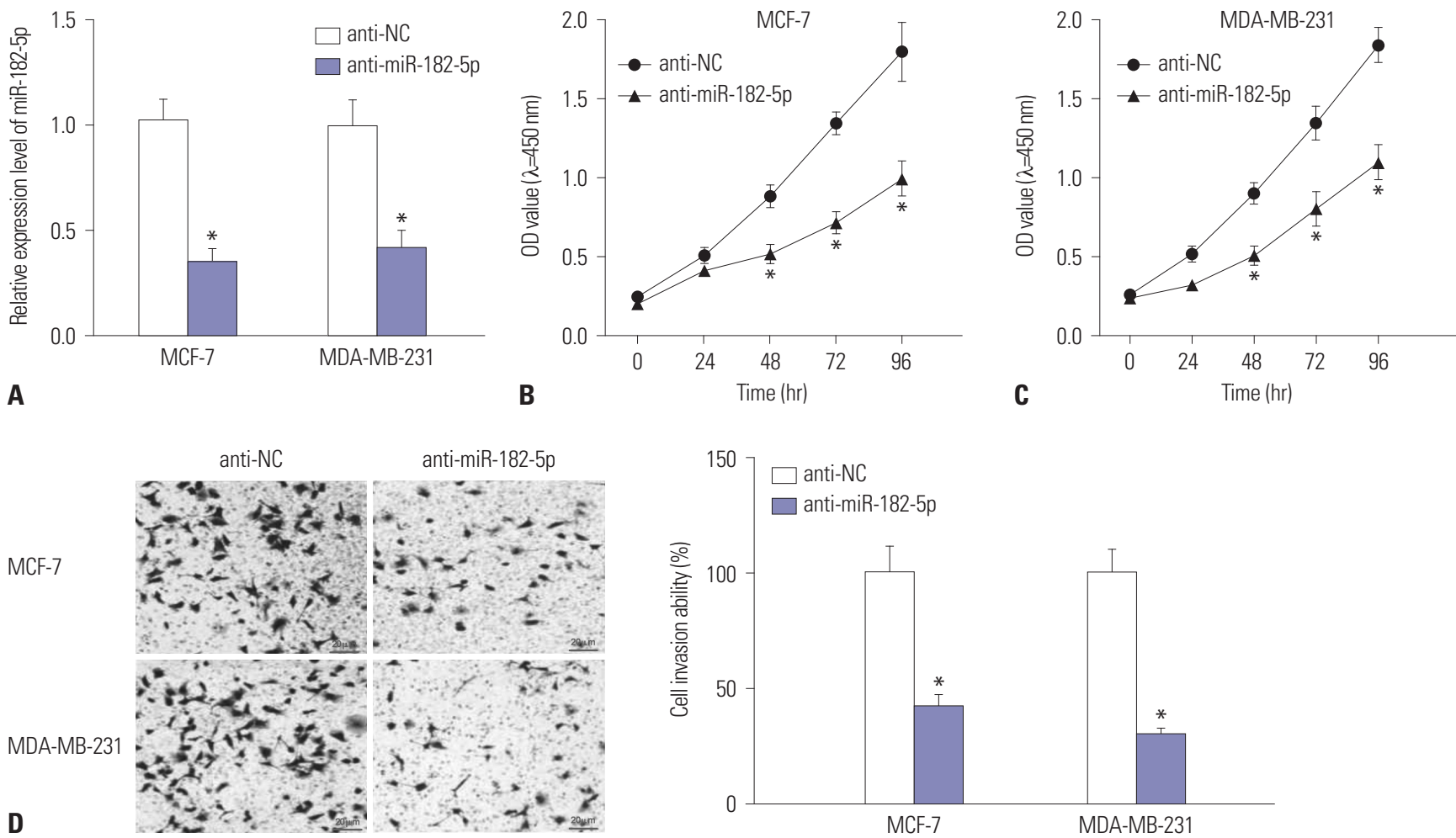

Fig. 2. Depletion of miR-182-5p inhibits cell proliferation and invasion of breast cancer cells. (A) Transfection efficacy was investigated in MCF-7 and MDA-MB-231 cells after transfection of anti-miR-182-5p or anti-NC. (B and C) The effect of anti-miR-182-5p on cell proliferation was investigated in MCF-7 and MDA-MB-231 cells by CCK-8. (D) The invasive ability was evaluated in MCF-7 and MDA-MB-231 cells transfected with anti-miR-182-5p or anti-NC. ${ }^{*} p<0.05$. 
cells (Fig. 2B and C). In addition, invasive ability was obviously impaired in both MCF-7 and MDA-MB-231 cells with the absence of miR-182-5p (Fig. 2D).

\section{PTEN is a direct target of miR-182-5p}

Generally, the function of miRNA is known to modulate targeted gene expression in various cancers. Therefore, a promising target of miR-182-5p was required for understanding the pathogenesis of BC. Bioinformatics analysis described the putative binding sites of miR-182-5p and 3'-UTR of PTEN (Fig. 3A). Hence, luciferase activity and Agol RIP assays were performed to validate the interaction. Results showed that overexpression of miR-182-5p leads to a marked reduction in luciferase activity in MDA-MB-231 and MCF-7 cells transfected with PTEN-WT, whereas its efficacy was lost in response to that in the PTEN-MUT group (Fig. 3B and C). However, absence of miR-182-5p showed the opposite effect on luciferase activity in MDA-MB-231 and MCF-7 cells (Fig. 3D and E). Moreover, the abundance of Agol-enriched PTEN was effectively enhanced in MCF-7 and MDA-MB-231 cells transfected with miR-182$5 \mathrm{p}$, compared with that in the miR-NC group; IgG failed to show any efficacy of enrichment (Fig. 3F). The abundance of PTEN was measured in BC tissues and cells by qRT-PCR and Western blot. The results revealed obvious decreases in the expression of PTEN in BC tissues and cells, compared with their corresponding control, respectively (Fig. 3G and H). Meanwhile, accumulation of miR-182-5p resulted in reductions in PTEN protein, while its abrogation induced elevated expression thereof, in MDA-MB-231 and MCF-7 cells (Fig. 3I).
hsa-miR-182-5p PTEN WT 3'UTR

A PTEN MUT 3'UTR

\section{3' UCACACU--CAAGAU----GGU--AACGGUUU 5' 5' ...GUCAUGGUUCAUGUGUUUCAGUUUGCCAAG... 3' 5' ...GUCAUGGUUCAUGUGUUUCAGUAACGgUUG... 3'}
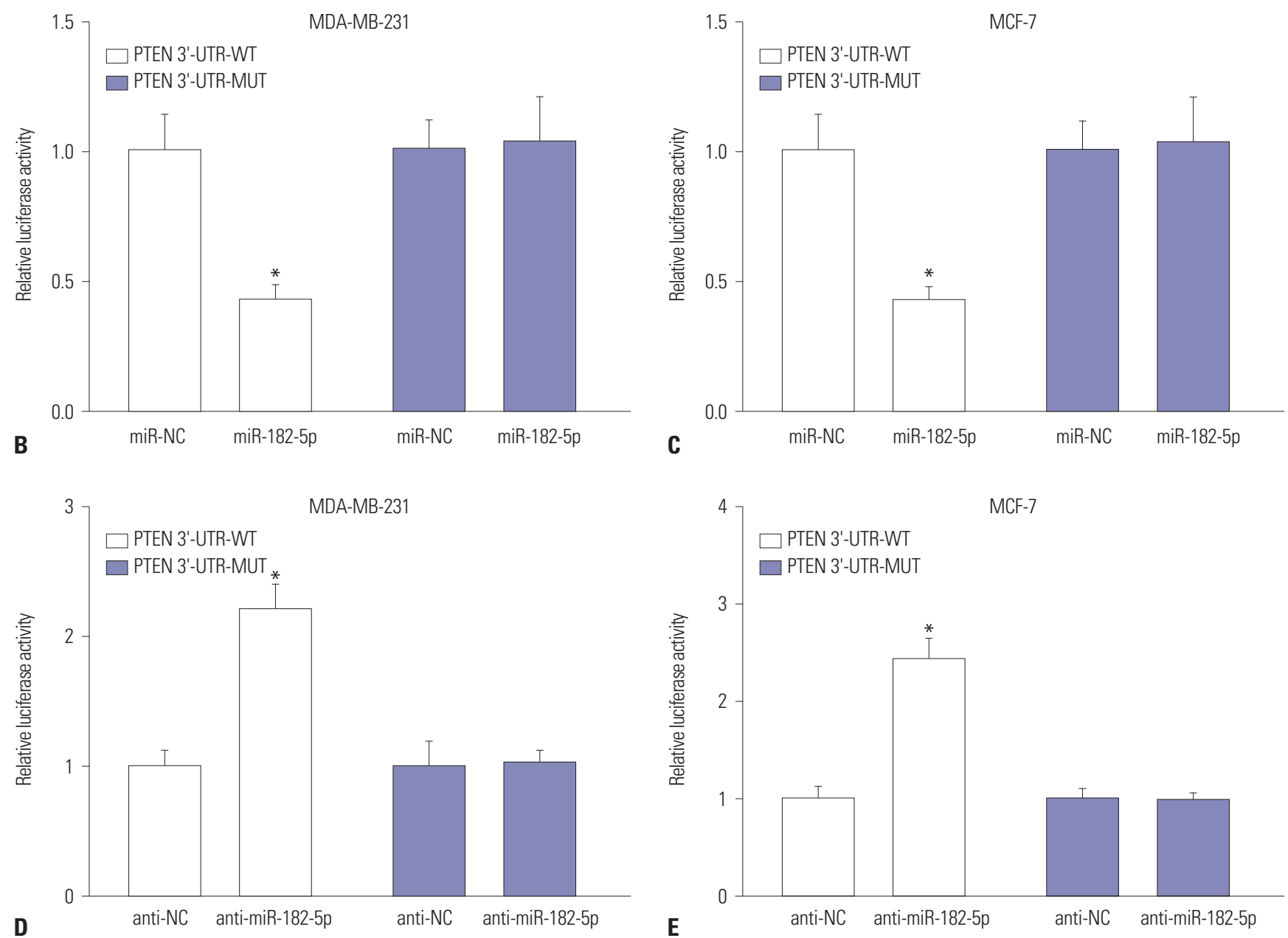

Fig. 3. PTEN a target of miR-182-5p. (A) Putative binding sites of miR-182-5p and PTEN was predicted by miRTarBase and miRanda. (B-E) The luciferase activity was investigated in MDA-MB-231 and MCF-7 cells co-transfected with WT or MUT luciferase vectors and miR-182-5p, anti-miR-182-5p or their negative control. ${ }^{*} p<0.05$. 

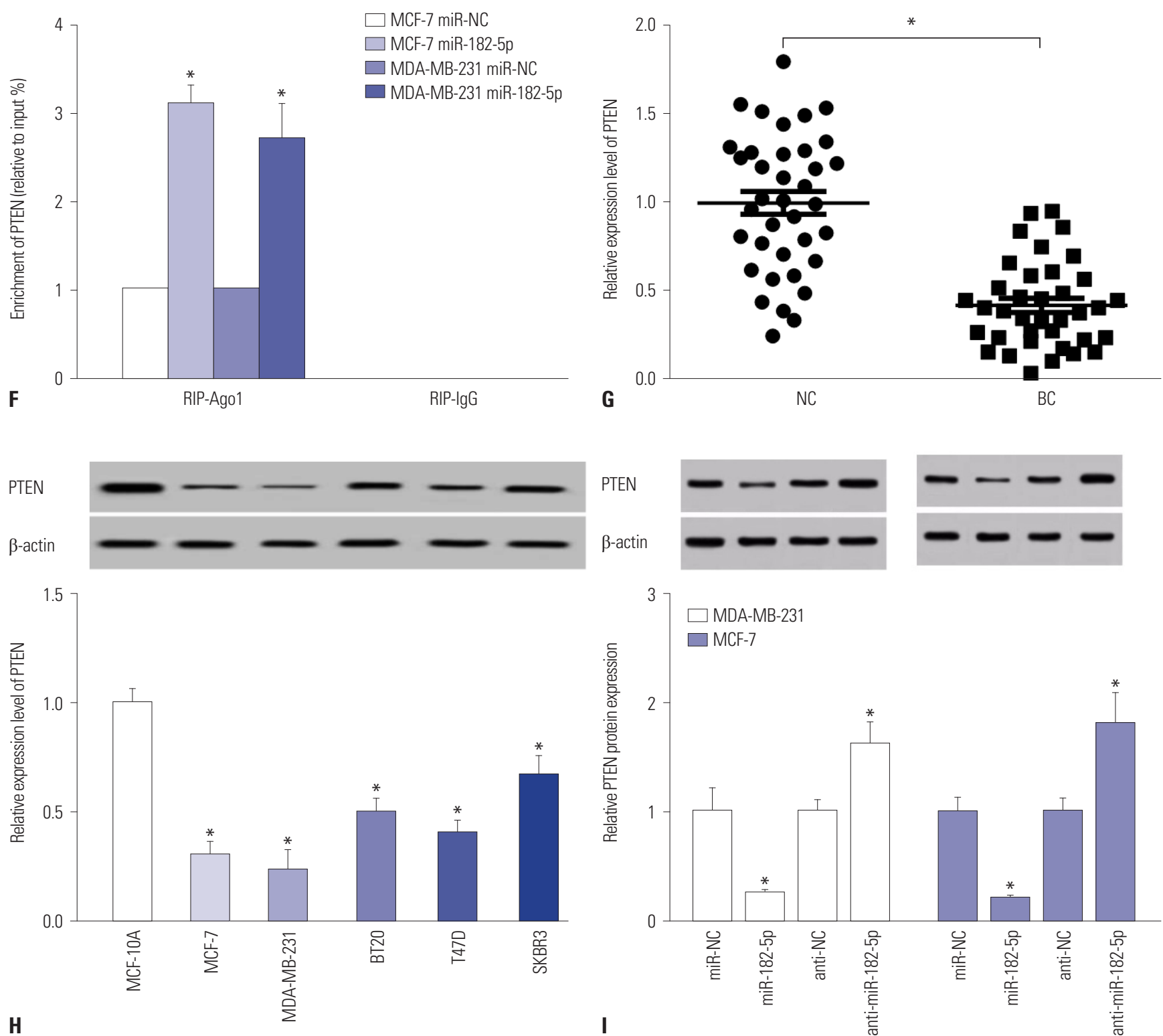

Fig. 3. PTEN a target of miR-182-5p. (F) The abundance of enrichment of PTEN was detected in MDA-MB-231 and MCF-7 cells after Ago1 RNA immunoprecipitation (RIP). (G and H) The expression of PTEN was measured in breast cancer (BC) tissues and cells at the mRNA and protein level, respectively. (I) The effect of miR-182-5p on PTEN protein expression was examined in MDA-MB-231 and MCF-7 cells. * $p<0.05$.

\section{Addition of PTEN ablates miR-182-5p-mediated promotion of cell proliferation and invasion}

Since PTEN was found to be a target of miR-182-5p, we further investigated whether PTEN was required for the miR182-5p-mediated effect on proliferation and invasion in BC cells. Unsurprisingly, the abundance of miR-182-5p was markedly elevated in MCF-7 and MDA-MB-231 cells with transfection of miR-182-5p mimics (Fig. 4A). Meanwhile, the mRNA expression of PTEN was evidently increased in cells suffering from transfection of PTEN overexpression vector (Fig. 4B). Moreover, introduction of PTEN overexpression vector reversed the inhibitory effect of miR-182-5p on expression of PTEN in MCF-7 and MDA-MB-231 cells (Fig. 4C). Functional analysis demonstrated that richness of miR-182-5p contributed to cell proliferation in MDA-MB-231 and MCF-7 cells, whereas restoration of PTEN attenuated the effect (Fig. $4 \mathrm{D}$ and E). Furthermore, invasive cells were greatly increased in MCF-7 and MDA-MB-231 cells transfected with miR-182$5 p$, compared with that in miR-NC group, while the presence of PTEN ablated the regulatory effect of miR-182-5p on cell invasion (Fig. 4F).

\section{MiR-182-5p exhaustion suppresses tumor growth in vivo}

Given that miR-182-5p deletion was found to protect against cell proliferation and invasion in BC cells, we next investigated whether miR-182-5p knockdown might inhibit tumor growth in vivo. Tumor size was effectively suppressed in anti-miR- 

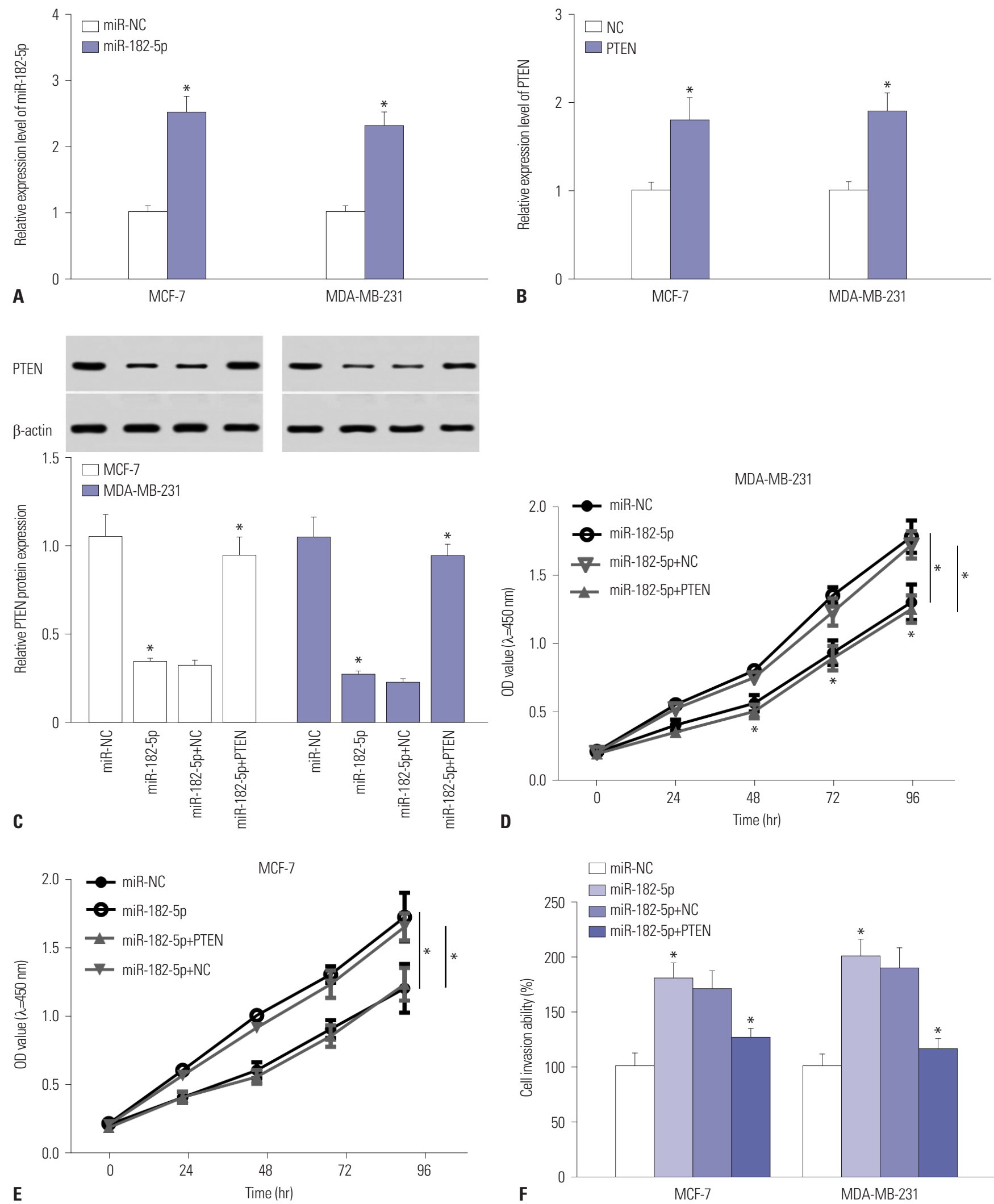

Fig. 4. Addition of PTEN reverses miR-182-5p-mediated promotion on cell proliferation and invasion of breast cancer cells. (A) The expression of miR182-5p was detected in MCF-7 and MDA-MB-231 cells transfected with miR-182-5p or miR-NC. (B) PTEN level was examined in MCF-7 and MDAMB-231 cells transfected with PTEN or NC. (C) The expression of PTEN was measured in MCF-7 and MDA-MB-231 cells co-transfected with miR182-5p and NC or PTEN. (D and E) The effect of miR-182-5p and PTEN on cell proliferation was investigated in MDA-MB-231 and MCF-7 cells. (F) The effect of miR-182-5p and PTEN on invasive ability was detected in MDA-MB-231 and MCF-7 cells by trans-well analysis. ${ }^{*} p<0.05$. 

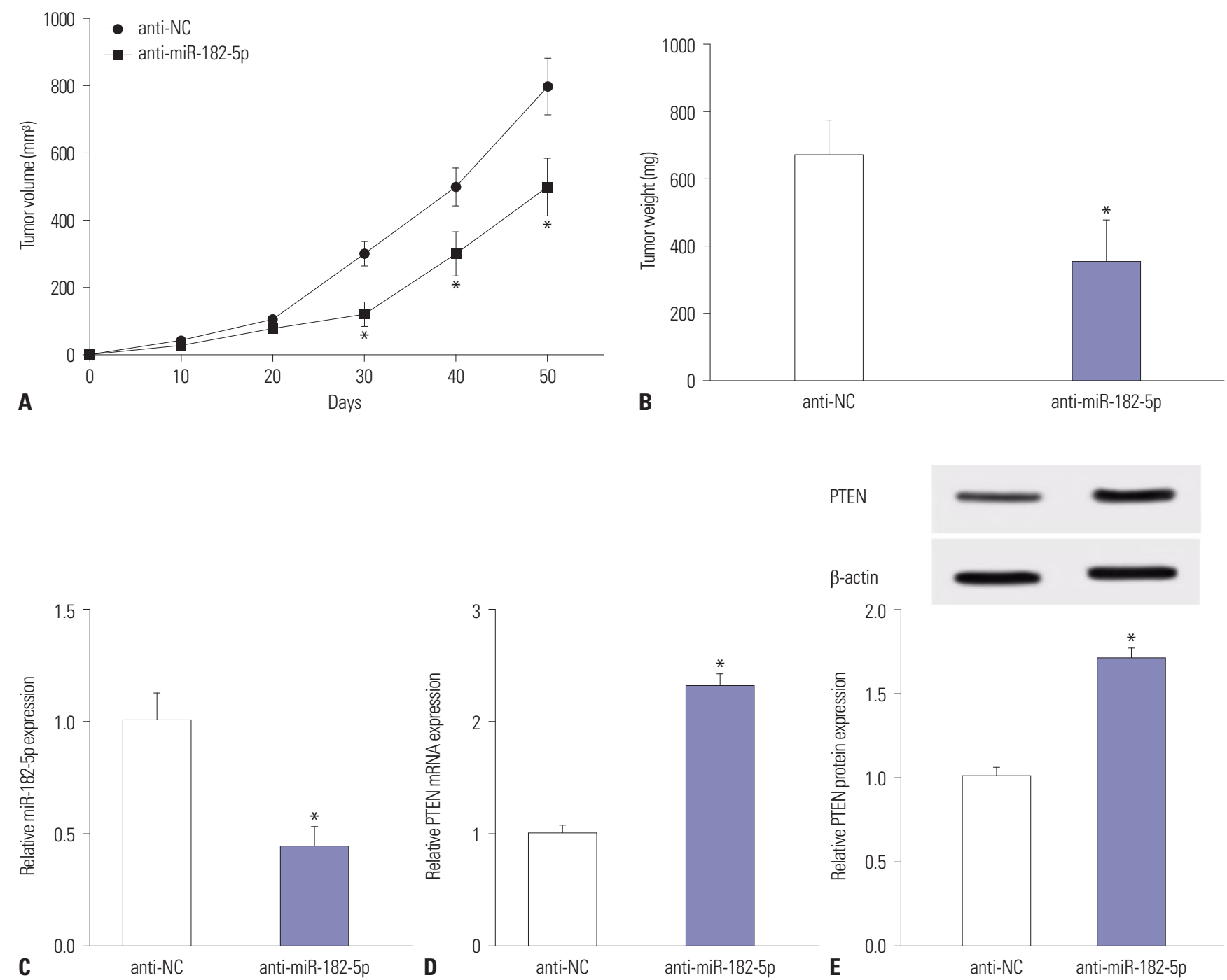

Fig. 5. MiR-182-5p deficiency suppresses MCF-7 xenograft tumor growth in vivo. (A) Tumor volume was examined every 10 days for five times after cell implantation. (B) Tumor weight was detected at the end point. (C) The expression of miR-182-5p was detected in tumor tissues. (D and E) The abundance of PTEN was evaluated in tumors by qRT-PCR and Western blot, respectively. ${ }^{*} p<0.05$.

182-5p treated mice, compared with the anti-NC group (Fig. 5A). Moreover, miR-182-5p deficiency led to a marked loss of tumor weight, compared with anti-NC treatment (Fig. 5B). Subsequently, the expression levels of miR-182-5p and PTEN were investigated in tumor tissues, revealing an obvious reduction of miR-182-5p in the tumors with anti-miR-182-5p insult (Fig. 5C). Besides, the expression of PTEN was enhanced at mRNA and protein levels in tumors in anti-miR-182-5p group, compared with the anti-NC group (Fig. 5D and E).

\section{DISCUSSION}

MiRNAs, as new biomarkers, play an important role in regulating invasion, proliferation, metastasis, apoptosis, and chemotherapy in BC. ${ }^{18}$ Here we found that miR-182-5p expression is enhanced in BC tissues and cells and predicted poor survival, which is consistent with previous work. ${ }^{19}$ Additionally, we explored the effect of miR-182-5p on cell proliferation and invasion and outlined interactions between miR-182-5p and PTEN in BC cells.

MiRNAs have been reported to serve as tumor suppressor or oncogene in $\mathrm{BC}$ progression. For example, miR-30 level was increased in $\mathrm{BC}$ and its knockdown blocked cell proliferation and invasive ability and increased survival time in mice with MCF-7 xenograft. ${ }^{20}$ Moreover, miR-27b protected against proliferation by altering cell metabolism by regulating pyruvate dehydrogenase complex (PDHX) in BC. ${ }^{21}$ Meanwhile, miR-433 was reported as a tumor suppressor implicated in cell proliferation, apoptosis, and the metastatic potential of BC. ${ }^{22}$ In this study, we demonstrated miR-182-5p exhaustion protected against cell proliferation and invasion of BC cells. This is in agreement with previous research that showed miR182-5p abrogation limits cell proliferation and results in 
apoptosis. $^{14}$

Potential target genes have been suggested to be required for functional miR-182-5p. For instance, miR-182-5p was suggested to play an essential role in hepatocellular carcinoma progression by regulating forkhead box O3a (FOXO3a). ${ }^{23}$ In addition, RAB27A was also reported as a functional target of miR-182-5p, which has an important impact on cell viability, migration, and invasion in gastric cancer. ${ }^{11}$ In the current study, we probed the interaction between miR-182-5p with PTEN and validated the inhibitory effect of PTEN on miR182-5p-mediated protective in $\mathrm{BC}$, indicating that PTEN might play a suppressive role in BC.

Emerging evidence suggests that various genes are associated with the risk of BC, including PTEN. ${ }^{24}$ PTEN has been reported as a target of miR-498 to regulate cell proliferation and migration of BC. ${ }^{16}$ Furthermore, PTEN has been found to limit proliferation and to induce apoptosis through miR-142-5p in BC. ${ }^{17}$ In addition, miR-21 was shown to be correlated with poor outcomes in $\mathrm{BC}$ patients and to regulate chemo-resistance and invasion via inhibiting PTEN expression in BC MCF7 cells. ${ }^{25}$ Meanwhile, miR-130a was suggested as an oncogenic target that inhibited PTEN abundance to promote cell proliferation and tumor growth in multiple cancers, including BC. ${ }^{26}$ These reports also supported our view of PTEN as a tumor suppressor. Similar to the in vitro results, absence of miR-182$5 \mathrm{p}$ inhibited tumor growth and enhanced PTEN expression in our xenograft model induced by MCF-7 cells: in vivo xenograft models are widely used in preclinical drug trials and BC studies. ${ }^{27,28}$ As the microenvironment is complex and metastases in vivo remain a challenge in $\mathrm{BC},{ }^{29,30}$ metastases need more in vivo investigation for $\mathrm{BC}$ treatment in the future.

In conclusion, miR-182-5p was elevated and correlated with poor survival in BC. MiR-182-5p deficiency lowered cell proliferation and invasion of BC cells. Intriguingly, PTEN was interacted with miR-182-5p and abated the promotive effect of miR-182-5p on BC progression. Moreover, miR-182-5p depletion impaired tumor growth in the xenograft model. These results indicated that miR-182-5p knockdown hindered cell proliferation and invasion by targeting PTEN in BC, providing a novel therapeutic approach for BC treatment.

\section{AUTHOR CONTRIBUTIONS}

Conceptualization: Yue-Sheng Zhao. Data curation: Yue-Sheng Zhao. Formal analysis: Yue-Sheng Zhao, Wei-Chao Yang. Investigation: Yue-Sheng Zhao, Wei-Chao Yang. Methodology: Wei-Chao Yang. Project administration: Hong-Wei Xin. Resources: Ji-Xia Han. Software: Hong-Wei Xin. Writing-original draft: Su-Gang Ma. Writingreview \& editing: Ji-Xia Han, Su-Gang Ma.

\section{ORCID iDs}

Yue-Sheng Zhao https://orcid.org/0000-0002-5438-3057 Wei-Chao Yang https://orcid.org/0000-0002-8552-9098
Hong-Wei Xin

Ji-Xia Han

Su-Gang Ma

https://orcid.org/0000-0002-9708-8226

https://orcid.org/0000-0003-4033-3718

https://orcid.org/0000-0002-6406-6063

\section{REFERENCES}

1. Siegel RL, Miller KD, Jemal A. Cancer statistics, 2018. CA Cancer J Clin 2018;68:7-30.

2. Varghese F, Wong J. Breast cancer in the elderly. Surg Clin North Am 2018;98:819-33.

3. Greenwood HI, Dodelzon K, Katzen JT. Impact of advancing technology on diagnosis and treatment of breast cancer. Surg Clin North Am 2018;98:703-24.

4. Mandujano-Tinoco EA, García-Venzor A, Melendez-Zajgla J, Maldonado V. New emerging roles of microRNAs in breast cancer. Breast Cancer Res Treat 2018;171:247-59.

5. O'Bryan S, Dong S, Mathis JM, Alahari SK. The roles of oncogenic miRNAs and their therapeutic importance in breast cancer. Eur J Cancer 2017;72:1-11.

6. Zhang N, Zhang H, Liu Y, Su P, Zhang J, Wang X, et al. SREBP1, targeted by miR-18a-5p, modulates epithelial-mesenchymal transition in breast cancer via forming a co-repressor complex with Snail and HDAC1/2. Cell Death Differ 2018 Jul 9 [Epub]. Available at: https://doi.org/10.1038/s41418-018-0158-8.

7. Yin C, Zhang G, Sun R, Pan X, Wang X, Li H, et al. miR1855p inhibits Factin polymerization and reverses epithelial mesenchymal transition of human breast cancer cells by modulating RAGE. Mol Med Rep 2018;18:2621-30.

8. Li C, Zhang J, Ma Z, Zhang F, Yu W. miR-19b serves as a prognostic biomarker of breast cancer and promotes tumor progression through PI3K/AKT signaling pathway. Onco Targets Ther 2018;11: 4087-95.

9. Croset M, Pantano F, Kan C, Bonnelye E, Descotes F, Alix-Panabières $\mathrm{C}$, et al. miRNA-30 family members inhibit breast cancer invasion, osteomimicry, and bone destruction by directly targeting multiple bone metastasis-associated genes. Cancer Res 2018;78:5259-73.

10. Guo J, Liu C, Wang W, Liu Y, He H, Chen C, et al. Identification of serum miR-1915-3p and miR-455-3p as biomarkers for breast cancer. PLoS One 2018;13:e0200716.

11. Li Y, Chen S, Shan Z, Bi L, Yu S, Li Y, et al. miR-182-5p improves the viability, mitosis, migration, and invasion ability of human gastric cancer cells by down-regulating RAB27A. Biosci Rep 2017;37:BSR20170136.

12. Hirata H, Ueno K, Shahryari V, Deng G, Tanaka Y, Tabatabai ZL, et al. MicroRNA-182-5p promotes cell invasion and proliferation by down regulating FOXF2, RECK and MTSS1 genes in human prostate cancer. PLoS One 2013;8:e55502.

13. Zhang K, Wang YW, Wang YY, Song Y, Zhu J, Si PC, et al. Identification of microRNA biomarkers in the blood of breast cancer patients based on microRNA profiling. Gene 2017;619:10-20.

14. Sharifi M, Moridnia A. Apoptosis-inducing and antiproliferative effect by inhibition of miR-182-5p through the regulation of CASP9 expression in human breast cancer. Cancer Gene Ther 2017;24:75-82.

15. Chen CY, Chen J, He L, Stiles BL. PTEN: tumor suppressor and metabolic regulator. Front Endocrinol (Lausanne) 2018;9:338.

16. Chai C, Wu H, Wang B, Eisenstat DD, Leng RP. MicroRNA-498 promotes proliferation and migration by targeting the tumor suppressor PTEN in breast cancer cells. Carcinogenesis 2018;39: 1185-96.

17. Xu W, Wang W. MicroRNA1425p modulates breast cancer cell 
proliferation and apoptosis by targeting phosphatase and tensin homolog. Mol Med Rep 2018;17:7529-36.

18. Bertoli G, Cava C, Castiglioni I. MicroRNAs: new biomarkers for diagnosis, prognosis, therapy prediction and therapeutic tools for breast cancer. Theranostics 2015;5:1122-43.

19. Krishnan K, Steptoe AL, Martin HC, Wani S, Nones K, Waddell N, et al. MicroRNA-182-5p targets a network of genes involved in DNA repair. RNA 2013;19:230-42.

20. Bao S, Wang X, Wang Z, Yang J, Liu F, Yin C. MicroRNA-30 mediates cell invasion and metastasis in breast cancer. Biochem Cell Biol 2018 Jun 12 [Epub]. Available at: https://doi.org/10.1139/ bcb-2018-0032.

21. Eastlack SC, Dong S, Ivan C, Alahari SK. Suppression of PDHX by microRNA-27b deregulates cell metabolism and promotes growth in breast cancer. Mol Cancer 2018;17:100.

22. Zhang T, Jiang K, Zhu X, Zhao G, Wu H, Deng G, et al. miR-433 inhibits breast cancer cell growth via the MAPK signaling pathway by targeting Rapla. Int J Biol Sci 2018;14:622-32.

23. Cao MQ, You AB, Zhu XD, Zhang W, Zhang YY, Zhang SZ, et al. miR-182-5p promotes hepatocellular carcinoma progression by repressing FOXO3a. J Hematol Oncol 2018;11:12.
24. Rousset-Jablonski C, Gompel A. Screening for familial cancer risk: focus on breast cancer. Maturitas 2017;105:69-77.

25. Dai X, Fang M, Li S, Yan Y, Zhong Y, Du B. miR-21 is involved in transforming growth factor $\beta 1$-induced chemoresistance and invasion by targeting PTEN in breast cancer. Oncol Lett 2017;14: 6929-36.

26. Wei H, Cui R, Bahr J, Zanesi N, Luo Z, Meng W, et al. miR-130a deregulates PTEN and stimulates tumor growth. Cancer Res 2017;77:6168-78.

27. Holen I, Speirs V, Morrissey B, Blyth K. In vivo models in breast cancer research: progress, challenges and future directions. Dis Model Mech 2017;10:359-71.

28. Rashid OM, Takabe K. Animal models for exploring the pharmacokinetics of breast cancer therapies. Expert Opin Drug Metab Toxicol 2015;11:221-30

29. Martins D, Schmitt F. Microenvironment in breast tumorigenesis: friend or foe? Histol Histopathol 2018:18021.

30. Kotecki N, Lefranc F, Devriendt D, Awada A. Therapy of breast cancer brain metastases: challenges, emerging treatments and perspectives. Ther Adv Med Oncol 2018;10:1758835918780312. 